\title{
Differential effects of specific amino acid restriction on glucose metabolism, reduction/oxidation status and mitochondrial damage in DU145 and PC3 prostate cancer cells
}

\author{
XIAOYI LIU, YA-MIN FU and GARY G. MEADOWS \\ Department of Pharmaceutical Sciences, College of Pharmacy, Washington State University, Pullman, WA 99164-6534, USA
}

Received October 7, 2010; Accepted December 30, 2010

DOI: $10.3892 / 01.2011 .237$

\begin{abstract}
Selective amino acid restriction targets mitochondria to induce apoptosis of DU145 and PC3 prostate cancer cells. Biochemical assays and flow cytometry were uitilized to analyze the glucose consumption, lactate production, pyruvate dehydrogenase (PDH), nicotinamide adenine dinucleotide (NAD)/ NADH and nicotinamide adenine dinucleotide phosphate (NADP)/NADPH ratios, mitochondrial glutathione peroxidase (GPx), manganese superoxide dismutase (SOD), glutathione, reactive oxygen species (ROS) and DNA damage in DU145 and $\mathrm{PC}$ prostate cancer cells cultured under various amino acid deprived conditions. Restriction of tyrosine and phenylalanine (Tyr/Phe), glutamine (Gln) or methionine (Met) differentially modulated glucose metabolism and PDH and antioxidant enzyme activity in the mitochondria of the two prostate cancer cell lines. In DU145 cells, Gln and Met restriction increased glucose consumption and decreased lactate production, but Tyr/ Phe restriction did not. The examined restrictions increased mitochondrial PDH activity and accumulation of ROS. Gln and Met restriction increased GPx activity. Tyr/Phe and Met restriction increased SOD during the first 2 days of the restriction, and the activity returned to the basal level on day 4 . All amino acid restrictions decreased reduced glutathione (GSH) and induced mitochondrial DNA damage. In PC3 cells, all amino acid restrictions reduced glucose consumption and lactate production. Gln restriction increased ROS and elevated GPx activity. Tyr/Phe restriction increased SOD activity. The amino acid restriction decreased GSH, but did not cause mitochondrial DNA damage. Specific amino acid dependency differentially regulates glucose metabolism, oxidation-reduction reactions of mitochondria and mitochondrial damage in DU145 and PC3 prostate cancer cell lines.
\end{abstract}

Correspondence to: Dr Gary G. Meadows, Department of Pharmaceutical Sciences, Box 646534, College of Pharmacy, Washington State University, Pullman, WA 99164-6534, USA

E-mail: meadows@wsu.edu

Key words: glucose metabolism, amino acid dependency, oxidative stress, mitochondrial damage, prostate cancer

\section{Introduction}

The metabolic abnormalities of prostate cancer cells have yet to be adequately investigated $(1,2)$. Results of our previous studies showed that relative specific amino acid dependency is one of the metabolic abnormalities of human prostate cancer cells. A significant effect of specific amino acid restriction is the induction of apoptosis in invasive, androgen-independent DU145 and PC3 prostate cancer cells, but not in normal human prostate epithelial cells (HPrE) or in non-invasive, androgen-dependent LNCaP prostate cancer cells $(3,4)$. Moreover, selective amino acid restriction targets the mitochondria of DU145 and PC3 cells. For example, the restriction of tyrosine and phenylalanine (Tyr/Phe), glutamine (Gln) or methionine (Met) inhibits ATP synthesis of the two cell lines (3). Additionally, the restriction of Tyr/Phe or Met in DU145 and Met in PC3 cells reduces the mitochondrial membrane potential (5).

Mitochondria trigger cell death in various ways, including the disruption of electron transport and energy metabolism, the release/activation of proteins that mediate apoptosis and the alteration of cellular reduction/oxidation (redox) potential (6). Specific amino acid restriction was previously found to alter the mitochondrial distribution of various apoptosis-related proteins, such as apoptosis-inducing factor, Bak and Bcl-XL, in DU145 cells (5). The present study was designed to determine whether specific amino acid restriction alters glucose metabolism and cellular redox status, and whether these alterations are related to mitochondrial DNA damage in DU145 and PC3 cells restricted for specific amino acids.

\section{Materials and methods}

Cell culture. Two human prostate cancer cell lines, DU145 and PC3, were maintained in suitable media (DMEM for DU145 and RPMI-1640 for PC3) with 10\% fetal bovine serum (Equitech-Bio, Inc., Kerrville, TX, USA). HPrE cells were cultured in PrEBM Basal Medium with PrEGM SingleQuot kit supplement and growth factors (Lonza Walkersville Inc., Walkersville, MD, USA). The cells were initially cultured in complete medium until they achieved $30-40 \%$ confluence prior to changing to the amino acid-depleted medium. For these experiments, cells were cultured in media depleted of individual amino acids (Met or Gln) or two combined amino 
acids (Tyr/Phe). The amino acid-deprived media were custommanufactured by Life Technologies (Grand Island, NY, USA), as previously described $(3,7)$. The experiments were repeated at least three times with similar results.

Determination of glucose consumption and lactate production. Cells $(10,000)$ were seeded in 12-well plates and cultured in complete or respective amino acid-free medium for 4 days. The glucose or lactate concentrations in the culture medium and cell numbers (Beckman Coulter Vi-Cell XR counter, Brea, CA, USA) were determined daily. Glucose consumption and lactate production rates were normalized to the cell number.

Mitochondrial pyruvate dehydrogenase activity. Pyruvate dehydrogenase $(\mathrm{PDH})$ catalyzes the reaction that converts pyruvate to acetyl-CoA, which then enters the citric acid cycle (TCA). Thus, this enzyme links glycolysis and the TCA cycle. The cells were cultured in an amino acid-deprived medium for 4 days. The attached cells were collected for mitochondria isolation on days 2 , 3 and 4 (Mitochondrial DNA Isolation kit; Biovision, Mountain View, CA, USA) and to determine PDH enzyme activity (MitoProfile microplate assay kit for PDH activity; MitoSciences Inc., Eugene, OR, USA). The isolation and assay procedures were performed according to the manufacturer's instructions and the results were normalized to the mitochondrial protein content.

Measurement of reactive oxygen species. Intracellular reactive oxygen species (ROS),including hydrogen peroxide and hydroxyl radicals, was detected with 5-(and-6)-chloromethyl-2',7'dichorodihydro-fluorescein diacetate, acetyl ester (Molecular Probes Inc., Eugene, OR, USA) staining and analyzed by flow cytometry. Briefly, $5 \mu \mathrm{M}$ of this compound was added into the culture medium $30 \mathrm{~min}$ prior to cell harvesting. The cells were then washed and resuspended in PBS. The fluorescence intensity of 10,000 events was analyzed with a FACScan flow cytometer (BD Biosciences, San Jose, CA, USA).

Determination of cellular nicotinamide adenine dinucleotide and nicotinamide adenine dinucleotide phosphate. Nicotinamide adenine dinucleotide (NAD)/NADH and nicotinamide adenine dinucleotide phosphate (NADP)/NADPH ratios were determined with NAD/NADH and NADP/NADPH quantification kits (Biovision) according to the manufacturer's instructions.

Glutathione peroxidase activity. Glutathione peroxidase (GPx) catalyzes the reduction of hydroperoxides, including hydrogen peroxide, by reduced glutathione (GSH) and protects the cell from oxidative damage. The enzyme activity during restriction of Tyr/Phe, Gln and Met was measured. The cells were cultured in specific amino acid-deprived medium for 4 days. The attached cells were collected on days 2, 3 and 4 and analyzed for GPx enzyme activity (Glutathione Peroxidase assay kit; Cayman Chemical Company, Ann Arbor, MI, USA). The results were normalized to the cellular protein content as determined by the Bio-Rad protein assay (Bio-Rad Laboratories Inc., Hercules, CA, USA).

Mitochondrial superoxide dismutase activity. Superoxide dismutase (SOD) catalyzes the conversion of superoxide anion into hydrogen peroxide and oxygen. The cells were treated with specific amino acid-deprived medium for 4 days and the attached cells were collected on days 2, 3 and 4 . The mitochondria were isolated (Mitochondrial DNA isolation kit; Biovision) and manganese (Mn) SOD enzyme activity was determined (SOD-560: A colorimetric assay kit for SOD activity; Applied Bioanalytical Labs, Sarasota, FL, USA). The isolation and assay procedures were performed according to the manufacturer's instructions. The results were normalized to the mitochondrial protein content as determined by the Bio-Rad protein assay (Bio-Rad Laboratories Inc.).

Measurement of reduced, oxidized and total glutathione. Glutathione is a signficant intracellular low-molecular weight thiol that plays a critical role in cellular defense against oxidative stress. Cells were cultured in amino acid-deprived medium for 4 days and the attached cells were collected to determine the amount of reduced glutathione (GSH), oxidized glutathione (GSSG) and total glutathione (Glutathione assay kit; Biovision) according to the manufacturer's instructions. The results were normalized to the cellular protein content as determined by the Bio-Rad protein assay (Bio-Rad Laboratories Inc.).

Measurement of mitochondrial DNA damage. Apurinic/ apyrimidinic (AP) sites are one of the major types of DNA lesions formed during the course of base excision and repair of oxidized or alkylated bases. The level of AP sites is a good indicator of DNA damage. Mitochondrial DNA (mtDNA) was isolated (Mitochondrial DNA isolation kit; Biovision) and quantified (Quant-It PicoGreen dsDNA reagent and kits; Molecular Probes Inc.). For each sample, $0.5 \mu \mathrm{g}$ mtDNA in triplicate was used to determine the density of AP sites. MtDNA AP site density was measured using a DNA damage quantification kit (Biovision). The procedures were performed according to the manufacturer's instructions.

Statistical analysis. The data in the figures are the means $\pm \mathrm{SE}$ from three separate experiments. The differences in the means were compared using one-way ANOVA. The curves were compared using two-way ANOVA. Multiple comparisons were performed by the least squares means method.

\section{Results}

Specific amino acid restriction differentially modulates glucose metabolism, cellular redox enzyme and the glutathione status in DU145 and PC3 prostate cancer cells. The data also indicate a correlation between the metabolic alterations and mitochondrial DNA damage in the two cell lines.

Amino acid restriction differentially modulates glucose consumption and lactate production in DU145 and PC3 cells. Fig. 1 shows the effects of amino acid restriction on glucose consumption and lactate production in DU145 and PC3 cells. Glucose consumption and lactate production did not change with the period of time that DU145 and PC3 cells were cultured in amino acid complete media. In DU145 cells, Gln and Met restriction increased glucose consumption, but glucose consumption was not significantly altered by the $\mathrm{Tyr} / \mathrm{Phe}$ restriction (Fig. 1A). Lactate production was 
A

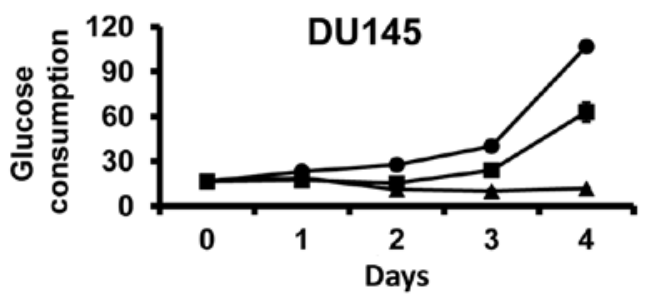

B

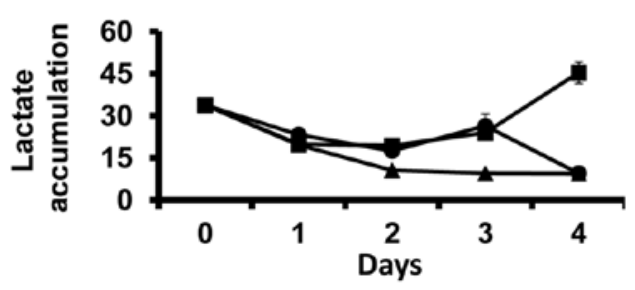

C
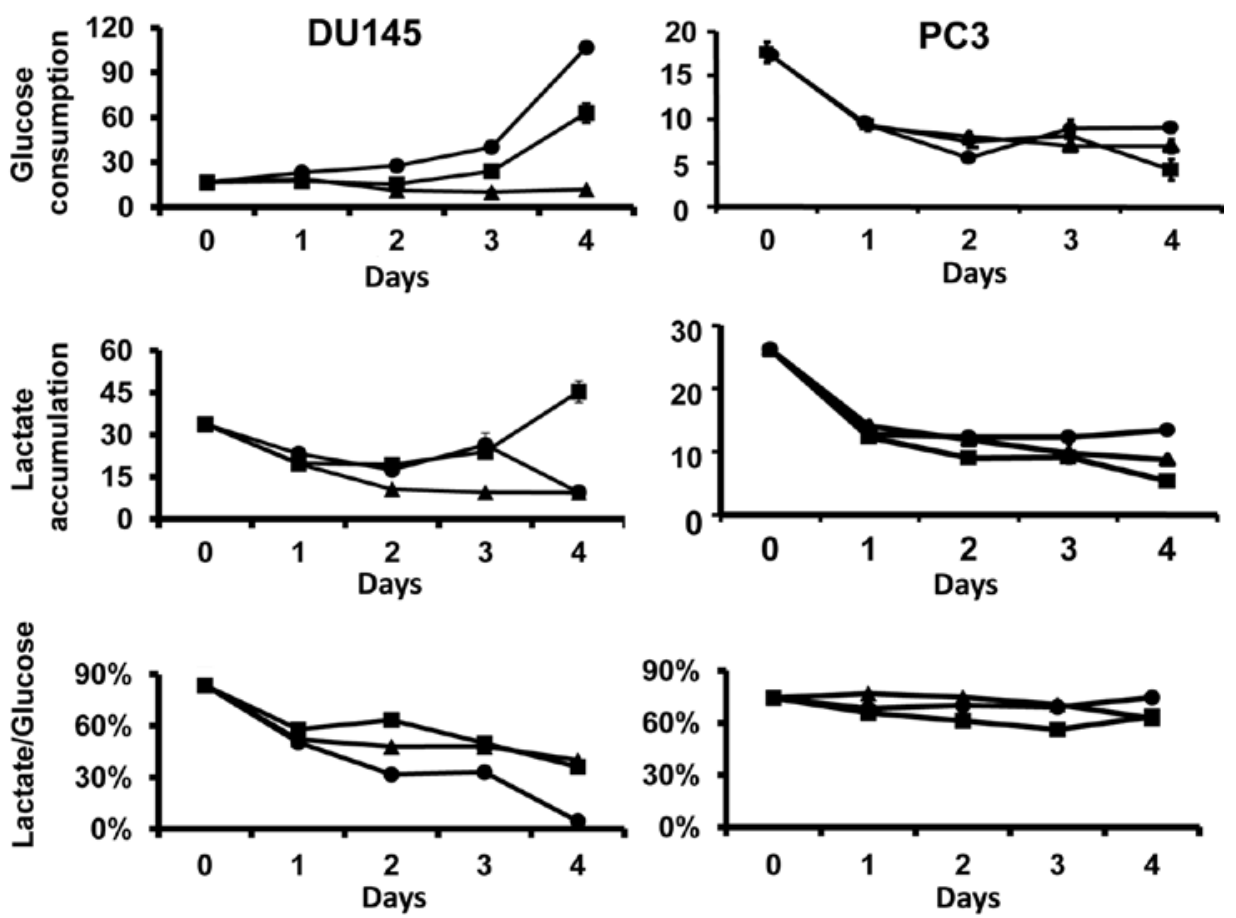

D

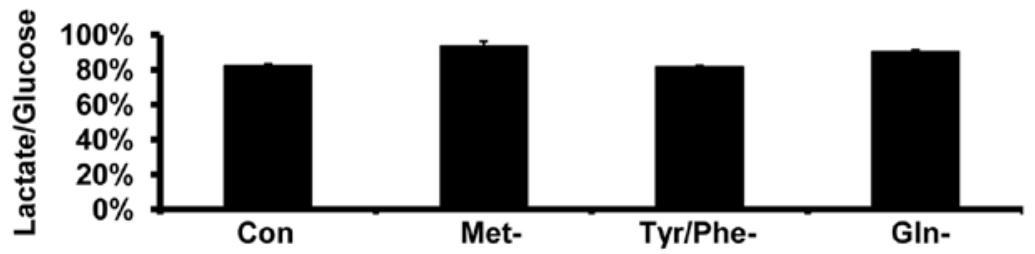

Figure 1. Effect of amino acid restriction on glucose metabolism. The cells were cultured with complete or amino acid-free medium for 4 days. The concentrations of glucose or lactate in the culture medium and the cell numbers were determined daily. Glucose consumption and lactate production rates were normalized to the cell number. The results are presented as the mean ( $\mu$ mol/10 $10^{6}$ cells/day) of three experiments \pm SE. (A) Amino acid restriction differentially modulated glucose consumption in DU145 and PC cells. In DU145 cells, the curves for glucose consumption varied for all amino acid restrictions (p<0.0001). Glutamine (Gln) restriction increased glucose consumption from days 1 to 4 ( $<<0.05)$. Methionine (Met) restriction increased glucose consumption only on day 4 ( $<<0.0001)$. Glucose consumption was slightly reduced by tyrosine and phenylalanine (Tyr/Phe) restriction from days 2 to 4 ( $<<0.05)$. In PC3, all amino acid restrictions reduced glucose consumption from days 1 to $4(\mathrm{p}<0.001)$. On day 4 , the Gln-restricted cells varied from the Met-restricted ones (p<0.05). The data in (A) were previously published (15). (B) Amino acid restriction differentially modulated lactate production in DU145 and PC3 cells. ANOVA analysis indicated a difference in the curves among the amino acid-restricted groups in DU145 cells $(\mathrm{p}<0.01)$. Lactate production was reduced by Tyr/Phe restriction from days 1 to 4 of restriction $(\mathrm{p}<0.01)$. Lactate production in Gln- and Met-deprived cells was also reduced during the first 2 days of restriction, and then returned to control levels on day 3. Lactate production was decreased in Gln-deprived cells and increased in Met-deprived cells on day 4 of restriction ( $<<0.05$ ). In PC3, amino acid restrictions reduced lactate production from days 1 to 4 ( $\mathrm{p}<0.001)$. (C) Lactate production/glucose consumption ratio of DU145 and PC3 cells. This ratio was calculated as lactate accumulation/2/glucose consumption $\mathrm{x} 100$. The lactate production/glucose consumption ratio was progressively reduced by the amino acid restrictions in DU145 cells. Amino acid restriction did not alter the ratio in PC 3 cells. (D) Lactate production/glucose consumption ratio of human prostate epithelial (HPrE). HPrE cells were cultured in complete or specific amino acid-deprived medium for 4 days. Lactate production/glucose consumption was not altered by amino acid restriction. $\mathbf{\bullet}$, Met restriction; $\mathbf{\wedge}$, Tyr/Phe restriction; $\bullet$, Gln restriction; Con, cells cultured in complete medium.

reduced by the Tyr/Phe restriction from days 1 to 4 of restriction. Lactate production in Gln- and Met-deprived cells was also reduced during the first 2 days of the restriction, but then returned to control levels on day 3. However, lactate production was decreased in Gln-deprived cells and increased in Met-deprived cells on day 4 (Fig. 1B). The lactate production/ glucose consumption ratio was reduced in DU145 cells by all specific amino acid restrictions at all time points (Fig 1C).

The specific amino acid restrictions reduced glucose consumption and lactate production in PC3 cells (Fig. 1A and $\mathrm{B}$ ). The lactate production/glucose consumption ratio was not altered by the amino acid restrictions (Fig. 1C).

Compared to DU145 and PC3 prostate cancer cells, the specific amino restrictions did not alter the glucose consumption or lactate production in HPrE cells (data not shown). This lack of alteration is due to the fact that the lactate production/ glucose consumption ratios did not differ in cells grown in amino acid complete medium (Fig. 1D).

$\mathrm{PDH}$ is the rate-determining enzyme for the entry of carbohydrate-derived acetyl units into oxidative metabolism (TCA cycle). PDH activity is negatively regulated by ATP and NADH. Cellular ATP is reduced in DU145 and PC3 cells during selective amino acid restriction (5), and the relative amount of NADH is also decreased by restriction. The mitochondrial activity of $\mathrm{PDH}$ was examined since $\mathrm{NADH}$ is a cofactor involved in PDH-mediated catalysis. In DU145 cells, the restrictions initially elevated PDH activity on day 2 of restriction, with the greatest increase in activity in Gln-restricted cells (Fig. 2A). The activity of PDH was not altered in PC3 cells by amino acid restriction (Fig. 2A). 

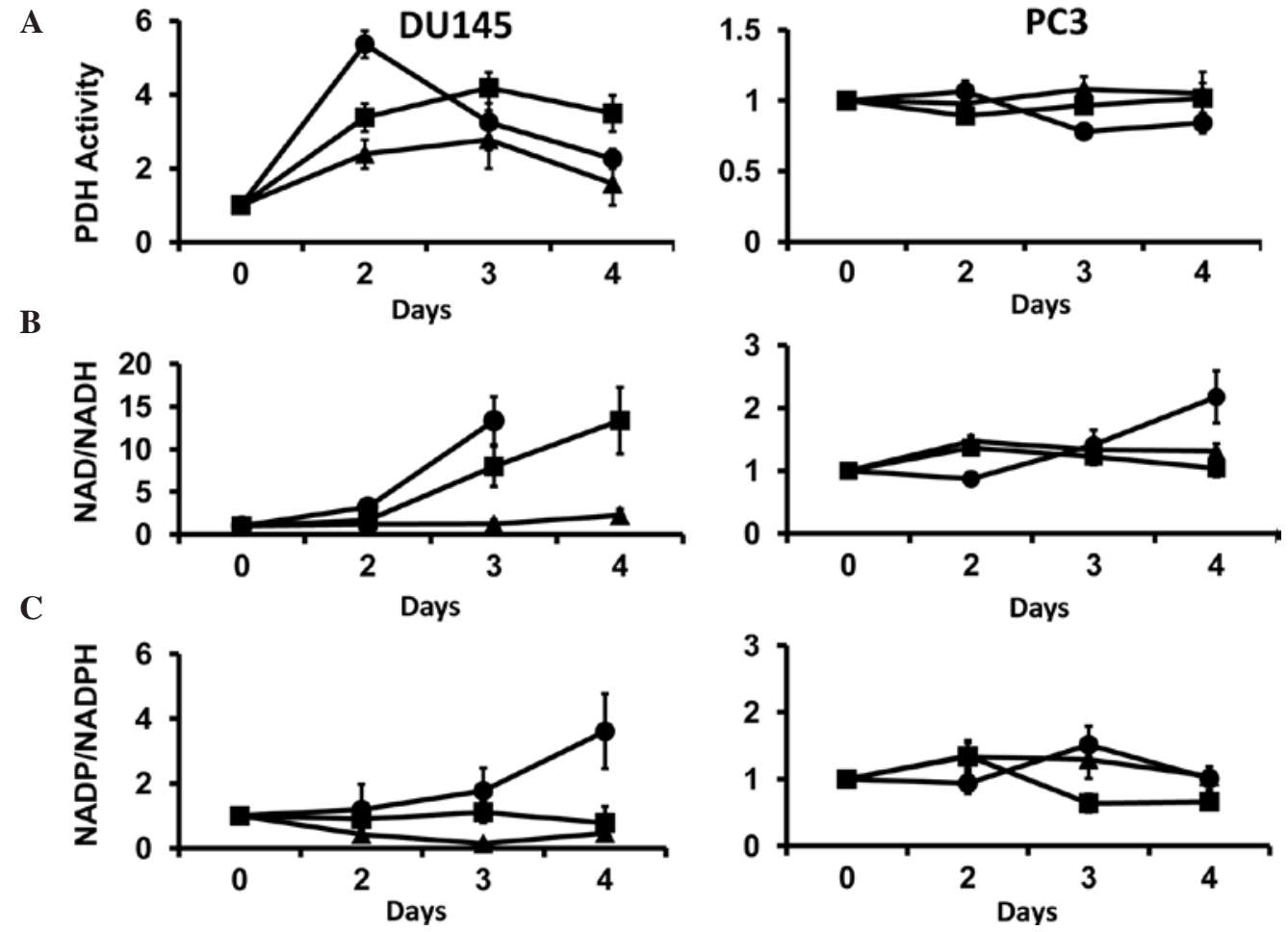

Figure 2. (A) Selective restriction of amino acids differentially modulated mitochondrial pyruvate dehydrogenase (PDH) activity in DU145 and PC3 cells. Data are expressed as the ratio of PDH activity in the amino acid-restricted cells compared to cells cultured in complete media (mean \pm SE). In DU145, the restrictions elevated PDH activity compared to the control after 2-3 days of restriction $(p<0.05)$. The activity of PDH was not significantly altered in PC3 cells by amino acid restriction compared to the control ( $\mathrm{p}>0.05$ ). (B) Cellular nicotinamide adenine dinucleotide (NAD)/NADH ratio. Data are expressed as the ratio of NAD/NADH in amino acid-deprived cells as compared to control. In DU145 cells, glutamine (Gln) (day 3) and methionine (Met) (days 3 and 4 ) restrictions elevated the NAD/NADH ratio $(\mathrm{p}<0.05)$. No ratio for Gln-deprived cells was noted on day 4, since NADH was undetectable on this day. Tyrosine and phenylalanine (Tyr/Phe) restriction did not alter the nicotinamide adenine dinucleotide phosphate (NADP)/NADPH ratio. In PC3 cells, the NAD/NADH ratio was only elevated on day $4(\mathrm{p}<0.05)$ and neither $\mathrm{Tyr} / \mathrm{Phe}$ nor Met restriction altered the ratio. (C) Cellular NADP/NADPH ratio. In DU145 cells, Gln restriction increased the ratio on day $4(\mathrm{p}<0.05)$. The ratio was not altered by Met-restriction and was reduced by Tyr/Phe restriction from days 2 to 3 . In PC 3 cells, the ratio was not significantly altered by the amino restrictions, with the exception of Met restriction, where the ratio was reduced on days 3 and 4 $(\mathrm{p}<0.05)$ compared to day $0 . \backsim$, Met restriction; $\boldsymbol{\wedge}, \mathrm{Tyr} / \mathrm{Phe}$ restriction; $\bullet$, Gln restriction.

Amino acid restriction differentially modulates reduction/ oxidation status in DU145 and PC3 cells. The NAD/NADH ratio increased in DU145 cells on days 3 and 4 in Gln- and Met-restricted cells (Fig. 2B). No data are available for day 4 for Gln-restricted cells, since NADH was not detectable on this day. The NADP/NADPH ratio also increased in Gln-restricted cells, reaching a maximum on day 4 (Fig. 2C). The NADP/NADPH ratio was reduced during $\mathrm{Tyr} / \mathrm{Phe}$ restriction throughout the experimental period (Fig. 2C), but was not changed by Met restriction.

In PC3 cells, the NAD/NADH ratio gradually increased to a maximum at 4 days during Gln restriction. The ratio was not significantly altered during Tyr/Phe or Met restriction (Fig. 2B). Moreover, the restrictions did not alter the NADP/ NADPH ratio (Fig. 2C) in these cells. These results show that selective amino acid restriction differentially alters the cellular redox status in PC3 and DU145 cells.

Amino acid restriction differentially modulates mitochondrial antioxidant enzymes and glutathione in DU145 and PC3 cells. ROS is mainly generated by the redox centers in the mitochondrial electron transport chain $(8,9)$. In DU145 cells, all the specific amino acid restrictions increased the amount of ROS. In PC 3 cells, only Met restriction increased the amount of ROS (Fig. 3A).
The clearance of ROS is closely regulated by the enzymes MnSOD and GPx in the mitochondria (8-13). The low molecular weight thiol, GSH, plays a key role in the cellular defense against oxidative stress $(9,14)$. The enzymatic action of GPx requires reduced GSH (9). Since cellular ROS is elevated in DU145 and PC3 cells, the activity of mitochondrial GPx and SOD and the amount of GSH in the two cell lines was examined. In DU145 and PC3 cells, the amino acid restrictions significantly increased mitochondrial GPx activity (Fig. 3B). In DU145 cells, the greatest increase was observed in Gln- and Met-restricted cells (Fig. 3B). In PC3 cells, the greatest increase was found in Met-restricted cells (Fig. 3B).

Tyr/Phe restriction increased mitochondrial SOD activity on day 2 in DU145 cells and the activity returned to control levels on day 4 (Fig. 3C). By day 4, SOD activity was lower than the control in the Met-restricted cells. Gln restriction did not alter mitochondrial SOD activity in DU145 cells (Fig. 3C). In PC3 cells, SOD activity was not significantly altered by Gln or Met restriction; however, it progressively increased in Tyr/ Phe-restricted cells over time (Fig. 3C). These results show that specific amino acid restriction differentially altered mitochondrial SOD and GPx activity in PC3 and DU145 cells.

Specific amino acid restriction reduced cellular GSH/GSSG in DU145 and PC3 cells (Fig. 3D). In DU145 cells, this ratio was similarly reduced by the amino acid restrictions. However, 
A

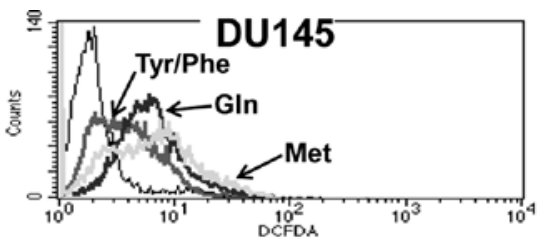

$\mathbf{B}$

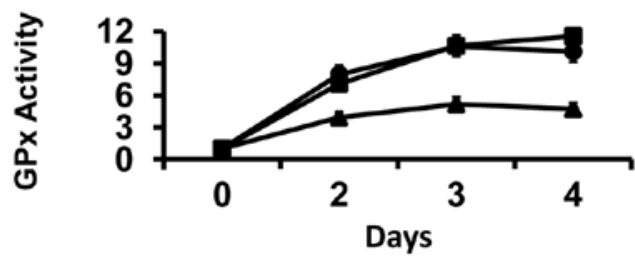

$\mathbf{C}$

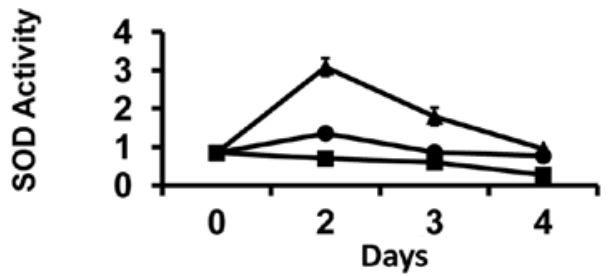

D

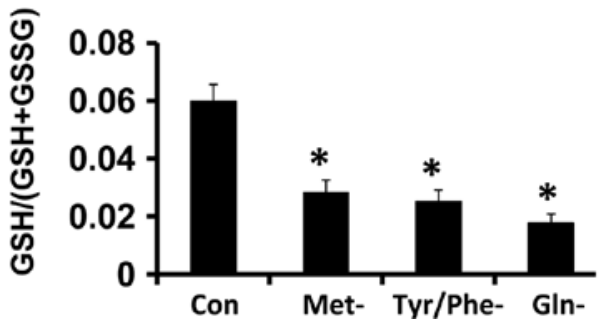

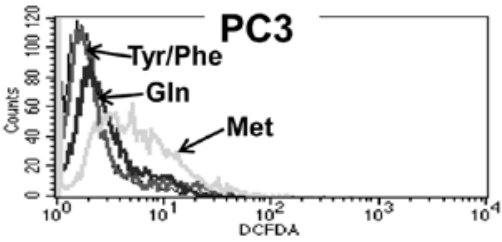
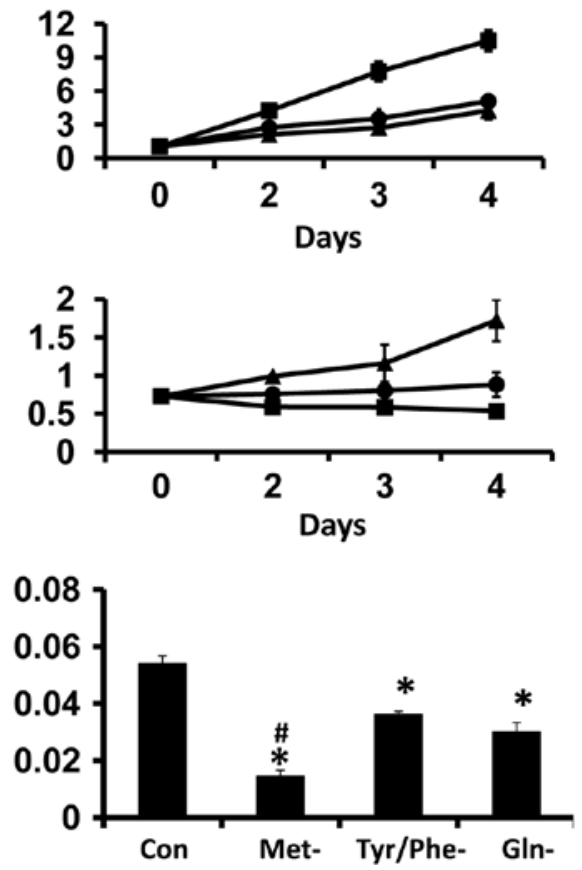

Figure 3. (A) Restriction of amino acids increased reactive oxygen species (ROS) in DU145 and PC3 cells. The cells were cultured in complete or amino aciddeprived media for 3 days and stained with dichorodihydro-fluorescein diacetate, acetyl ester. The x-axis shows the density of fluorescence. The y-axis shows the cell count. Peak shifts to the right are the cell population whose ROS increased. Tyr/Phe, Tyr/Phe-deprived; Met, Met-deprived, Gln, Gln-deprived. (B) Selective restriction of amino acids differentially increased cellular glutathione peroxidase (GPx) activity in DU145 and PC3 cells. GPx activity was expressed as nmol/min/mg protein. In DU145, the restrictions progressively increased GPx activity compared to day 0 (p<0.05). The increase was greatest in the glutamine (Gln)- and methionine (Met)-deprived cells compared to tyrosine and phenylalanine (Tyr/Phe)-deprived cells (p<0.001). GPx activity also progressively increased in PC3 cells $(\mathrm{p}<0.05)$; however, the increase was most pronounced in Met-deprived cells compared to Gln- or Tyr/Phe-restricted cells $(\mathrm{p}<0.00)$. (C) Selective restriction of amino acids differentially modulated mitochondrial superoxide dismutase (SOD) activity in DU145 and PC3 cells. SOD activity was expressed as U/ $\mu \mathrm{g}$ mitochondrial protein. In DU145 cells, Tyr/Phe restriction increased mitochondrial SOD activity by 3 -fold on day 2 of the restriction $(\mathrm{p}<0.001)$. The activity gradually returned to control levels by day 4 . Met restriction slightly reduced SOD activity on day 4 ( $\mathrm{p}<0.05$ ) compared to day 0 , and Gln restriction did not alter SOD activity. In PC3 cells, only Tyr/Phe restriction increased mitochondrial SOD activity and the difference was significant on days 3 to 4 (p<0.05). (D) Selective restriction of amino acids decreased the ratio of reduced glutathione (GSH) to total glutathione. The cells were cultured in complete or respective amino acid-deprived medium for 4 days and then the amount of GSH and total glutathione was determined. The amino acid deprivations reduced GSH in the two cell lines ( ${ }^{*} \mathrm{p}<0.05$ compared to Con). In PC3, a marked decrease was induced by Met restriction $\left({ }^{\#} \mathrm{p}<0.05\right.$ compared to the other amino acid restriction groups). Con, cells cultured in complete medium; $\mathbf{\square}$, Met restriction; $\boldsymbol{\Delta}$, Tyr/Phe restriction; $\bullet$, Gln restriction.

in the PC3 cells, the degree of reduction in GSH/GSSG in Met-restricted cells was 2-fold greater than that in Tyr/Phe- and Gln-restricted cells (Fig.3D). Collectively, the results in Fig. 3 indicate that specific amino acid restriction differentially modulates the cellular antioxidant system in DU145 and PC3 cells.

Amino acid restriction induces mitochondrial DNA damage in DU145 cells. The abnormal accumulation of ROS in cells could result in oxidative damage to mitochondrial nucleic acids. Mitochondrial DNA damage was observed in all amino acidrestricted DU145 cells (Fig. 4). Specific amino acid restriction decreased mitochondrial DNA damage in PC3 cells (Fig. 4).

\section{Discussion}

In previous studies, we found that relative specific amino acid dependency is one of the metabolic abnormalities of human prostate cancer cells that regulates cell proliferation and survival (3). These studies showed that selective amino acid restriction promotes mitochondrial outer membrane permeabilization and reduces intracellular ATP in DU145 and PC3 prostate cancer cells (5). Results of the present study showed that specific amino acid restriction differentially modulates cellular metabolism in the two cell lines (15), including glucose metabolism, cellular redox status and antioxidant systems. The increased glycolytic activity (Warburg effect) of cancer cells enhances the proliferation and resistance of the cells to apoptosis (16). We previously found that the addition of glucose or pyruvate differentially reduces or postpones the apoptotic effect of specific amino acid restriction in DU145 and PC3 cells (15).

In the present study, glucose metabolism was found to be differentially modulated by the specific restriction of amino acids in the two cell lines. For example, Gln and Met restriction increased glucose consumption in DU145 cells, but was not 


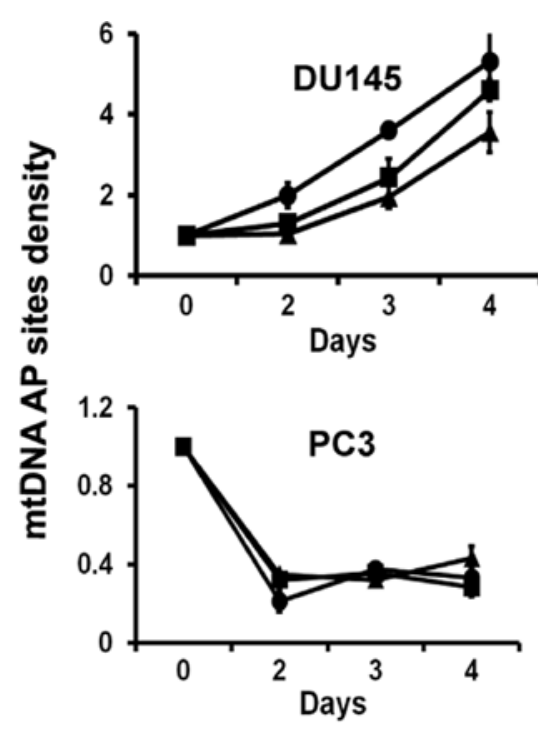

Figure 4. Selective amino acid restriction induced mitochondrial DNA damage in DU145 cells and decreased damage in PC3 cells. The cells were treated with specific amino acid-deprived medium for 4 days and the mitochondrial DNA (mtDNA) was determined on days 2 to 4 . The y-axis expresses the density of mtDNA apurinic/aprimidinic (AP) sites as a fold change compared to cells grown in complete medium (mean \pm SE of triplicate wells). Density is defined as the number of AP sites $/ 10^{5} \mathrm{bp}$. In DU145 cells, the site density increased progressively during amino acid restriction $(\mathrm{p}<0.05$ compared to day 0 ). The density of the AP sites decreased in PC3 with the amino acid restrictions from days 2 to 4 ( $\mathrm{p}<0.05$ compared to day 0$)$. $\mathbf{}$, Met restriction; $\boldsymbol{\Delta}$, Tyr/Phe restriction; $\bullet$, Gln restriction.

altered in Tyr/Phe-restricted cells (Fig. 1A). In DU145, the lactate production/glucose consumption ratio was reduced by the examined amino acid restrictions (Fig. 1C). Since the lactate production/glucose consumption ratio expresses the proportion of glycolysis relative to total glucose utilization, these findings suggest that selective amino acid restriction accelerates TCA cycle activity. PDH was the rate-determining enzyme for the entry of carbohydrate-derived acetyl units into oxidative metabolism, and the specific amino acid restrictions elevated the activity of this enzyme (Fig. 2A). These findings further support the hypothesis that the specific amino acid restriction accelerates the TCA cycle in DU145 cells. During the initial phase of specific amino acid restriction, a new energy balance is established in the cell that favors survival. However, this balance changes over time in such a manner that the continued pressure of amino acid restriction results in cell death, as previously described (3).

Since a variety of amino acids enter the TCA cycle in a variety of manners, the restriction of each amino acid may have different effects on the cycle. For example, Gln is a significant energy source in general and particularly for malignant cells. It is normally metabolized by L-glutamate dehydrogenase to $\alpha$-ketoglutarate, a substrate in the TCA cycle. Gln restriction is likely to reduce the availability of $\alpha$-ketoglutarate in the TCA cycle, leading to a compensatory accelerated conversion of pyruvate into acetyl-CoA, the primary component of the TCA cycle. This possibility is consistent with the results in Fig. 3A, where Gln restriction elicited a rapid increase of PDH activity in DU145 cells.

Compared to DU145 cells, the amino acid restrictions reduced glucose consumption and lactate production in
PC3 cells (Fig. 1A and B). The lactate production/glucose consumption ratio and $\mathrm{PDH}$ activity were not altered by the specific amino acid restrictions (Figs. 1C and 3A). The results indicate that the amino acid restrictions uniformly decreased glucose metabolism in PC3 cells. Therefore, during the initial phase of amino acid restriction, the cells adapted by gradually decreasing glucose metabolism, thereby establishing a new energy balance that favored cell survival.

The accelerated TCA cycle of DU145 cells by amino acid restriction alters the cellular redox status. This is expressed by fluctuations in the cellular NAD/NADH and NADP/ NADPH ratios and in the amount of ROS (Fig. 2 and 3). The alterations initially stimulated two antioxidant enzymes, SOD and GPx, to rid the cell of ROS (Fig. 3B and C). However, continued exposure to specific amino acid restriction resulted in the inability of the cells to compensate effectively and they subsequently died $(3,5,15)$. Additional evidence to support this finding is that GSH availability was limited (Fig. 3D) and mitochondrial DNA was damaged (Fig. 4) in addition to the damage induced in mitochondrial membranes associated with the induction of apoptosis $(3,5,15)$.

Compared to DU145, PC3 cells appear to have stronger antioxidant activity, since mitochondrial DNA is not damaged and the NAD/NADH and NADP/NADPH ratios are not altered to the same degree as the ratios in DU145 cells. Although all of the amino acid restrictions decrease GSH, Met-restricted cells showed the greatest decrease in GSH and in the accumulation of ROS (Fig. 3A and D). These data along with our previous finding may demonstrate why only Met restriction induces apoptosis in PC3 cells $(3,5,15)$.

In conclusion, findings of the present study show that specific amino acid restriction has a wide-spread effect on the metabolism of DU145 and PC3 prostate cancer cells, and it differentially modulates glucose metabolism and cellular redox status. Restriction of any amino acid causes metabolic stress in cells. The gradual reduction in intracellular amino acid levels during restriction is the proximal event that triggers signaling events to inhibit cell proliferation and induce cell death in prostate cancer cells $(3,5)$. The data obtained in this study correlate the metabolic alterations in DU145 and PC3 cells to mitochondrial damage and induction of apoptosis. However, additional metabolomic studies in these prostate cancer cells are required to further define which specific metabolic reactions are directly responsible for damaging mitochondria in the two prostate cancer cell lines during selective amino acid restriction. Since mitochondrial defects exist in cancer and mtDNA mutations in prostate cancer play a key role in cellular metabolism and behaviors $(17,18)$, this study provides a strong rationale for conducting additional studies to determine the manner in which selective amino acid restriction modulates specific mitochondrial functions in this type of cancer. Consequently, the mechanisms associated with specific amino acid dependency of prostate cancer cells may be elucidated, resulting in the development of novel therapeutic approaches.

\section{Acknowledgements}

This study was supported by funds from NIH/NCI R01 CA101035 (to G.G.M.). 


\section{References}

1. Tenniswood M: Apoptosis, tumour invasion and prostate cancer. Br J Urol 79: 27-34, 1997.

2. Denmeade SR, Isaacs JT, August JT, et al: Activation of programmed (apoptotic) cell death for the treatment of prostate cancer. In: Advances in Pharmacology (Vol 35). Academic Press, Inc., San Diego, CA, pp281-305, 1996.

3. Fu YM, Yu ZX, Li YQ, et al: Specific amino acid dependency regulates invasiveness and viability of androgen-independent prostate cancer cells. Nutr Cancer 45: 60-73, 2003.

4. Fu YM, Yu ZX, Lin H, Fu X and Meadows GG: Selective amino acid restriction differentially affects the motility and directionality of DU145 and PC3 prostate cancer cells. J Cell Physiol 217: 184-193, 2008.

5. Fu YM, Zhang H, Ding M, et al: Selective amino acid restriction targets mitochondria to induce apoptosis of androgen-independent prostate cancer cells. J Cell Physiol 209: 522-534, 2006.

6. Green DR and Reed JC: Mitochondria and apoptosis. Science 281: 1309-1312, 1998.

7. Fu YM, Yu ZX, Pelayo BA, et al: Focal adhesion kinasedependent apoptosis of melanoma induced by tyrosine and phenylalanine deficiency. Cancer Res 59: 758-765, 1999.

8. Nemoto S, Takeda K, Yu ZX, et al: Role for mitochondrial oxidants as regulators of cellular metabolism. Mol Cell Biol 20: 7311-7318, 2000

9. Balaban RS, Nemoto $\mathrm{S}$ and Finkel T: Mitochondria, oxidants, and aging. Cell 120: 483-495, 2005.
10. Arbiser JL: Molecular regulation of angiogenesis and tumorigenesis by signal transduction pathways: evidence of predictable and reproducible patterns of synergy in diverse neoplasms. Semin Cancer Biol 14: 81-91, 2004.

11. Wallace DC: Mitochondrial diseases in man and mouse. Science 283: 1482-1488, 1999.

12. Galluzzi L, Larochette N, Zamzami N, et al: Mitochondria as therapeutic targets for cancer chemotherapy. Oncogene 25: 4812-4830, 2006.

13. Matoba S, Kang JG, Patino WD, et al: 553 regulates mitochondrial respiration. Science 312: 1650-1653, 2006

14. Benlloch M, Mena S, Ferrer P, et al: Bcl-2 and Mn-SOD antisense oligodeoxynucleotides and a glutamine-enriched diet facilitate elimination of highly resistant B16 melanoma cells by tumor necrosis factor-alpha and chemotherapy. J Biol Chem 281: 69-79, 2006.

15. Fu YM, Lin H, Liu X, : Cell death of prostate cancer cells by specific amino acid restriction depends on alterations of glucose metabolism. J Cell Physiol 224: 491-500, 2010.

16. Weber G: Ordered biochemical program of gene expression in cancer cells. Biochemistry 66: 1164-1173, 2001.

17. Petros JA, Baumann AK, Ruiz-Pesini E, et al: mtDNA mutations increase tumorigenicity in prostate cancer. Proc Natl Acad Sci USA 102: 719-724, 2005.

18. Carew JS and Huang P: Mitochondrial defects in cancer. Mol Cancer 1: 9, 2002. 